\title{
Seroprevalence of Leptospirosis in a Tertiary Care Hospital - A Retrospective Study
}

\author{
Rama Gupta, Deepinder Chhina, Pooja Suri \\ Department of Microbiology, \\ Dayanand Medical College \& Hospital, Ludhiana, India
}

\begin{abstract}
Leptospirosis is a zoonotic disease with worldwide distribution. There is paucity of data about prevalence of this disease in North India. Thus, this study was undertaken to estimate the seroprevalence of leptospirosis in our region. A total of 3179 blood samples from patients suspected of leptospirosis were subjected to IgM ELISA (PanBio). The seroprevalence of leptospirosis in our area was found to be $20.7 \%$.
\end{abstract}

Keywords: IgM ELISA, Leptospirosis, Seroprevalence

\section{INTRODUCTION}

Leptospirosis is an anthropozoonotic infection of universal distribution caused by the pathogenic Leptospira spp. ${ }^{1}$ It is an acute febrile illness with a wide spectrum of clinical symptoms ranging from mild flu like illness to a variety of clinical syndromes including hepato-renal involvement with jaundice, severe pulmonary hemorrhage, myocarditis and meningitis. ${ }^{2}$ Leptospirosis is frequently under-diagnosed, due to non-specific symptoms early in the course of the disease and the difficulty of performing both, the culture and microscopic agglutination test (MAT), the reference serological test, which require experienced personnel. ${ }^{3}$ Detection of IgM antibodies by enzyme linked immunosorbent assay (ELISA) is now widely used for the diagnosis of leptospirosis, which is simple, easy to perform and can be used in routine.

In a countrywide study conducted by the National Reference Centre, Regional Medical Research Centre (ICMR), Port Blair, during the period 2000-2001, a seropositivity rate ranging from 0 to $46.8 \%$ among all cases of fever was observed from various parts of India. ${ }^{4}$ There is paucity of data about prevalence of this disease in North India. The present study was conducted with the objective of estimating the seroprevalence of leptospirosis in our area.

\section{Corresponding Author :}

Dr Rama Gupta

Associate Professor

Department of Microbiology,

Dayanand Medical College \& Hospital, Ludhiana, India

Email: ramagupta1404@yahoo.com

\section{MATERIALS AND METHODS}

\section{Blood Samples}

Blood samples from 3179 patients with suspicion of leptospirosis from various wards and ICU's of DMC\&H, Ludhiana during April 2010 - March 2012 were received in the Department of Microbiology. Serum from all the samples was separated and stored at $4^{\circ} \mathrm{C}$ till further processing.

\section{IgM ELISA Test}

All the serum samples were processed using Leptospira IgM ELISA (PanBio, Australia) as per the manufacturer's instructions. The absorbance of positive control, negative control and cut-off calibrator provided in the kit was used for the calculation of PanBio units. Samples were recorded as positive if the number of PanBio units were more than 11 .

\section{RESULTS}

This retrospective study was conducted on 3179 human subjects suspected of leptospirosis. Serum samples with a value greater than 11 PanBio units were taken as positive. Out of the 3179 samples, 657 $(20.7 \%)$ were positive for anti leptospiral antibodies by IgM ELISA. These 657 positive cases included 551 males and 106 females indicating a male preponderance. Figure I depict year-wise distribution of leptospira cases. Maximum number of cases were observed in the age group of $>50$ years $(31.5 \%)$ followed by $41-50 \mathrm{yr}(29.1 \%) ; 31-40 \mathrm{yr}(22.5 \%)$. Approximately $65 \%$ of the cases were between 21 and 50 years of age (Table I). 
Table I

Demographic profile of IgM positive leptospirosis cases

\begin{tabular}{lcccccc}
\hline & \multicolumn{2}{c}{$\mathbf{2 0 1 0 - 1 1}$} & $\mathbf{2 0 1 1 - 1 2}$ & Total & $\begin{array}{c}\text { \% Positivity } \\
(\mathbf{n = 6 5 7})\end{array}$ \\
\hline Age (years) & Male & Female & Male & Female & & \\
\hline $0-10$ & 2 & 0 & 0 & 0 & 2 & 0.3 \\
$11-20$ & 11 & 3 & 13 & 3 & 30 & 4.6 \\
$21-30$ & 22 & 17 & 35 & 10 & 84 & 13.0 \\
$31-40$ & 51 & 11 & 75 & 9 & 146 & 22.5 \\
$41-50$ & 81 & 4 & 89 & 14 & 188 & 29.1 \\
$>50$ & 92 & 19 & 80 & 16 & 207 & 31.5 \\
Total & $\mathbf{2 5 9}$ & $\mathbf{5 4}$ & $\mathbf{2 9 2}$ & $\mathbf{5 2}$ & $\mathbf{6 5 7}$ & \\
\hline
\end{tabular}

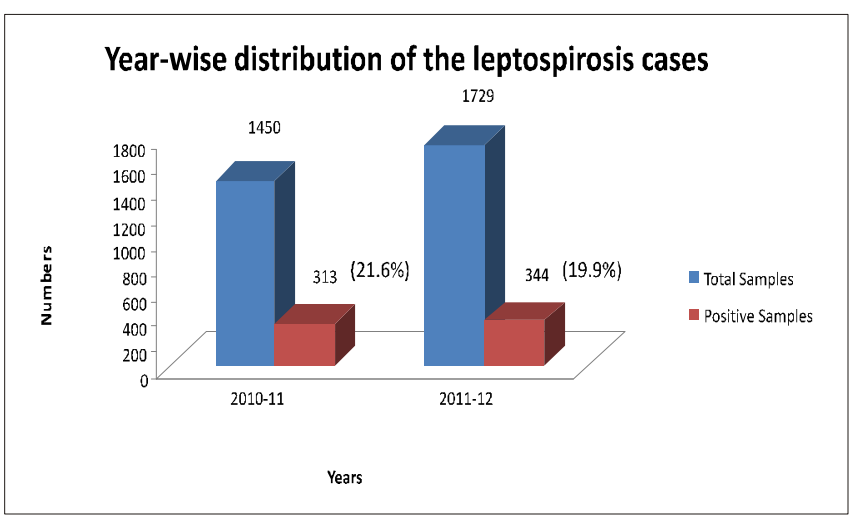

Fig. 1 : Year-wise distribution of the leptospirosis cases

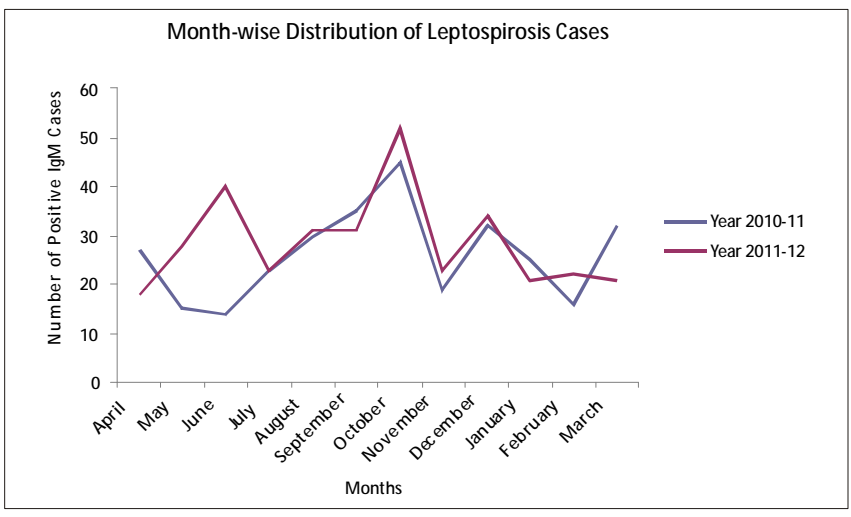

Fig. 2 : Month-wise distribution of the leptospirosis cases

Figure II shows the month-wise distribution of leptospirosis cases. Maximum numbers of cases were observed during the month of October in both the years.

\section{DISCUSSION}

Leptospirosis is an emerging global public health problem. In a 5 year retrospective study, Sethi et $a l^{5}$ reported an increased incidence of leptospirosis in Chandigarh (11.7\% in 2004 to $20.5 \%$ in 2008). Increasing seropositivity of leptospirosis was also observed by Sagar et $a l^{6}$ from Ludhiana (1.76\% in 2003 to $14.47 \%$ in 2006). In the present study seropositivity of leptospirosis was found to be $20.7 \%$. In contrast to the reports from North India, seroprevalence varying from $37 \%-57.5 \%$ have been reported from various centres in South India. ${ }^{7-9}$ The reason for low seroprevalence in North India may be because of the fact that this part of India receives less rainfall as compared to coastal regions and South.

In the present study, approximately $65 \%$ of the cases were seen in occupationally active age group (21-50 yrs) with male preponderance which is similar to the findings of Shekatkar et al \& Mathur et al. ${ }^{10}$

In the present study maximum numbers of cases were observed during the month of October in both the years. In this part of the country maximum rainfall is observed during the monsoon season between July and September. The increase in cases of leptospirosis during the monsoon and post monsoon period has also been reported in various studies. ${ }^{3,10}$

To conclude, the increase in leptospirosis cases during the last few years is possibly the result of greater awareness of this disease in North and drier part of the country. This study however is based on IgM ELISA 
and conclusive data can only be obtained by isolation and typing of various serovars.

Conflict of interest: None declared.

\section{REFERENCES}

1. Rao RS, Gupta N, Bhalla P, Agarwal SK. Leptospirosis in India and the rest of the world. Braz J Infect Dis 2003;7:178-93.

2. Effler PV, Bogard AK, Domen HY, Katz AR, Higa HY, Sasaki DM. Evaluation of eight rapid screening tests for acute leptospirosis in Hawaii. J Clin Microbiol 2002;40: 1464-69.

3. Maskey M, Shastri JS, Saraswathi K, Surpam R, Vaidya N. Leptospirosis in Mumbai: Post-Deluge Outbreak 2005. Indian J Med Microbiol 2006;24(4):337-8.

4. Christopher AM, Mohapatra AK, Binay, Dutta TK, Kanungo R, Das AK. Pulmonary manifestations of leptospirosis. In: Souvenir, 3rd Annual Conference of Indian Leptospirosis Society, Pondicherry, 2002.32.
5. Sethi S, Sharma N, Kakkar N, Taneja J, Chatterjee SS, Banga SS, et al. Increasing trends of leptospirosis in Northern India: A clinico-Epidemiological study. PLoS Negl Trop Dis. 2010; 4(1):e579. Published online 2010 January 12. doi: 10.1371/ journal.pntd.0000579.

6. Sagar V, Berry V. Increasing Seropositivity of Leptospirosis in a Medical College in Ludhiana. Indian J Publ Health 2008;52:50.

7. Shekatkar SB, Harish BN, Menezes GA, Parija SC. Clinical and serological evaluation of Leptospirosis in Puducherry, India. J Infect Dev Ctries 2010;4(3):139-43.

8. Jeyakumar N, Khan AA, Dhasarathan P, Singh A. Seroprevalence of leptospirosis in clinically suspected cases in Chennai city. Indian J Med Microbiol 2004;22:67.

9. Koteeswaran A. Seroprevalence of leptospirosis in man and animals in Tamilnadu. Indian J Med Microbiol 2006;24(4):32931.

10. Mathur M, De A, Turbadkar D. Leptospirosis Outbreak in 2005: L.T.M.G. Hospital Experience. Indian J Med Microbiol 2009;27(2):153-5. 


\section{PDF created with pdfFactory Pro trial version www.pdffactory.com}

